\section{Treatment strategy for pediatric giant mucinous cystadenoma: A case report}

\author{
Shun Watanabe, 1,2 \\ Shunsuke Nagashima, ${ }^{1}$ Chihiro Onagi, ${ }^{1}$ \\ Nobuto Yamazaki, ${ }^{1}$ Shuhei Shimada, ${ }^{1}$ \\ Masato Sakai, ${ }^{1}$ Shun Yanai, ${ }^{3}$ \\ Yoichi Haga, ${ }^{3}$ Akira Ohara, ${ }^{3}$ \\ Minoru Kuroiwa1 \\ 1Department of Surgery, Toho \\ University School of Medicine, Tokyo; \\ 2First Department of Surgery, Dokkyo \\ Medical University, Tochigi; \\ 3Department of Pediatrics, Toho \\ University School of Medicine, Tokyo, \\ Japan
}

\begin{abstract}
Because of their rarity, the treatment strategy for pediatric ovarian epithelial tumors is controversial, especially for a giant cystadenoma. We report the largest mucinous cystadenoma (MCA) case in the pediatric literature thus far. A 12-year-old girl had abdominal distention and visited our hospital. She had a multilocular cyst with some protuberance on the inside and high values of CA 19-9 and CA-125. We diagnosed her with a left MCA and performed a left oophorectomy. The tumor was the stage IA borderline malignant MCA and weighed $11.8 \mathrm{~kg}$. Five years have passed, the patient has not experienced recurrence or metastasis. The resection of giant tumors can affect respiration and circulation. However, pre- or intra-operative drainage may lead to dissemination and adhesion. When we treat pediatric giant ovarian epithelial tumors, we must understand the findings that suggest the possibility of malignancy to decide appropriately as to whether drainage should be performed.
\end{abstract}

\section{Introduction}

Ovarian tumors are estimated to occur in 2.6 per 100,000 girls younger than 15 years of age, representing less than $2 \%$ of all tumors in children. ${ }^{1}$ MCA (Mucinous Cystadenoma) and SCA (Serous Cystadenoma) arise from epithelial elements and are rare pediatric ovarian tumors. Ovarian epithelial tumors account for only $10-17 \%$ of these tumors. 2,3 Most ovarian epithelial tumors are cystadenomas, $75 \%$ of which are serous and $25 \%$ of which are mucinous..$^{4,5}$

We report the case of a 12-year-old female patient with a large MCA. The tumor weighed $11800 \mathrm{~g}$, which is the largest MCA in the pediatric literature. The treatment strategy for giant ovarian epithelial tumors has sometimes been controversial. Herein, we discuss the treatment of a pediatric MCA, especially a giant MCA.

\section{Case Report}

A 12-year-old girl had severe abdominal distention with increasing abdominal girth that had started 6 months earlier. She visited our hospital because her abdominal distention worsened despite her diet restriction.

Although she had menarche 9 months prior to presentation, her second menstruation had not come. Additionally, she had never experienced sexual relations.

The girl was $170 \mathrm{~cm}$ tall and weighed $63 \mathrm{~kg}$. Her body mass index was 21.8. She had no fever or abdominal pain. Her abdomen was markedly distended without fluctuation.

Abdominal X-ray showed elevated diaphragms and a lesion occupying a large space in the abdominal cavity. Her routine blood analysis and renal functions were normal: white blood cell count, $4.40 \times 10^{3} / \mathrm{mm}^{3}$ (4.3-10.8 × 103); hemoglobin, $11.1 \mathrm{~g} / \mathrm{dL}$ (12-18 g/dL); hematocrit, 34.3\% (35-53\%); platelet count, $220 \times 103 / \mu \mathrm{L}(150-400 \times$ $103 / \mu \mathrm{L})$; alanine transaminase, 13 units/L (5-40 units/L); aspartate aminotransferase, 22 units/L (5-42 units/L); urea nitrogen, 16 $\mathrm{mg} / \mathrm{dl}(8-20 \mathrm{mg} / \mathrm{dL})$; and creatinine, 0.87 $\mathrm{mg} / \mathrm{dL}(0.4-1.0 \mathrm{mg} / \mathrm{dL})$

The values of tumor markers were as follows: hCG (Human Chorionic Gonadotropin), $<0.003$ units $/ \mathrm{mL}(0-5$ units $/ \mathrm{mL}) ; \alpha$-fetoprotein, $0.7 \mathrm{ng} / \mathrm{mL}(<5$ $\mathrm{ng} / \mathrm{mL})$; carcinoembryonic antigen, 117.5 ng/mL (0-4.60 ng/mL); CA 19-9, 5029.0 units $/ \mathrm{mL}(<39$ units $/ \mathrm{mL})$; and CA-125, 96.3 units $/ \mathrm{mL} \quad(0-35$ units $/ \mathrm{mL})$. The patient's CA 19-9 and CA-125 levels were high.

Abdominal US (Ultrasonography) showed a giant multilocular cystic lesion that had some solid lesions, which protruded into the inside of the giant lesion. There was an oviduct-like structure on the dorsal side (Figure 1). T2-weighted MRI (Magnetic Resonance Imaging) also showed multilocular, high-intensity cysts with a stained-glass-like structure with a distinct septum and various concentrations of internal solution. Solid lesions had a lower intensity than that of the internal
Correspondence: Shun Watanabe, Department of Surgery, Toho University School of Medicine, 611-1 Omori-Nishi, Ota-ku, Tokyo, 143-8541, Japan.

Tel.: +81.03-3762-4151 - Fax: +81.03-3298-4348. Email: shun-wat@dokkyomed.ac.jp

Key words: Pediatric surgery, Ovarian cyst, Mucinous cystadenoma, Borderline malignancy.

Contributions: the authors contributed equally.

Conflict of interest: the authors declare no potential conflict of interest.

Funding: none.

Received for publication: 3 June 2019.

Revision received: 5 August 2019.

Accepted for publication: 5 August 2019.

This work is licensed under a Creative Commons Attribution NonCommercial 4.0 License (CC BY-NC 4.0).

(C) Copyright: the Author(s), 2019

Licensee PAGEPress, Italy

Pediatric Reports 2019; 11:8190

doi:10.4081/pr.2019.8190

solution on T2-weighted images. On the bottom of the dorsal cyst wall, there was a structure that appeared to be an oviduct. Bilateral hydronephroses were caused by cyst oppression (Figure 2). There was no finding suggesting peritoneal distant metastasis with MRI or CT (Computed Tomography).

We diagnosed a multilocular cyst connecting to the oviduct-like feature as an ovarian cystadenoma. Therefore, we performed a laparotomy.

We approached her abdomen by a lower abdominal horizontal incision and submitted her transparent ascites for cytology. The left oviduct was stretched and connected to the left lower side of the cyst. Although the border of the oviduct and tumor was clear, that of the ovarian parenchyma and tumor was ambiguous (Figure 3A,B). Therefore, we performed left oophorectomy. There were no findings that caused us to suspect membrane damage or lymph node metastasis or distant metastasis or dissemination in the peritoneal cavity. We thought the tumor was benign or borderline malignant. Therefore, we decided to preserve the oviduct. We ligated a thickened vessel entering the tumor from the mesometrium and removed the tumor. Adhesion to other organs was mild. Ascites cytology indicated that the tumor not malignant, and the intraoperative section diagnosis was diagnosed as an MCA. We sutured each layer of the 
abdominal wall and finished the operation.

The multilocular cystic tumor was 420 $\times 220 \times 200 \mathrm{~mm}$ and $11.8 \mathrm{~kg}$ (Figure 3C,D). It contained mucinous fluid, and the luminal surface was covered with a single layer of endocervical-like epithelial cells. In some areas, nuclear stratification into 2 or 3 layers and the formation of reactive granulation tissue were observed pathologically. Nuclear fission, high nuclear atypia, and stromal invasion were not observed. Therefore, we diagnosed her tumor as an FIGO (International Federation of Gynecology and Obstetrics) stage IA

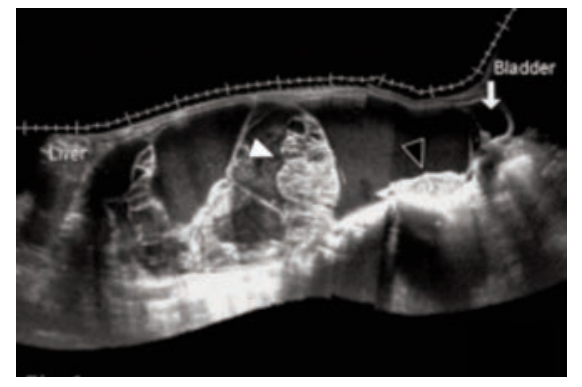

Figure 1. Preoperative combined image of US. There was a multilocular cyst with some solid lesions (white arrowhead) protruding into the inside. There was an oviduct-like structure on the dorsal side (black arrowhead).

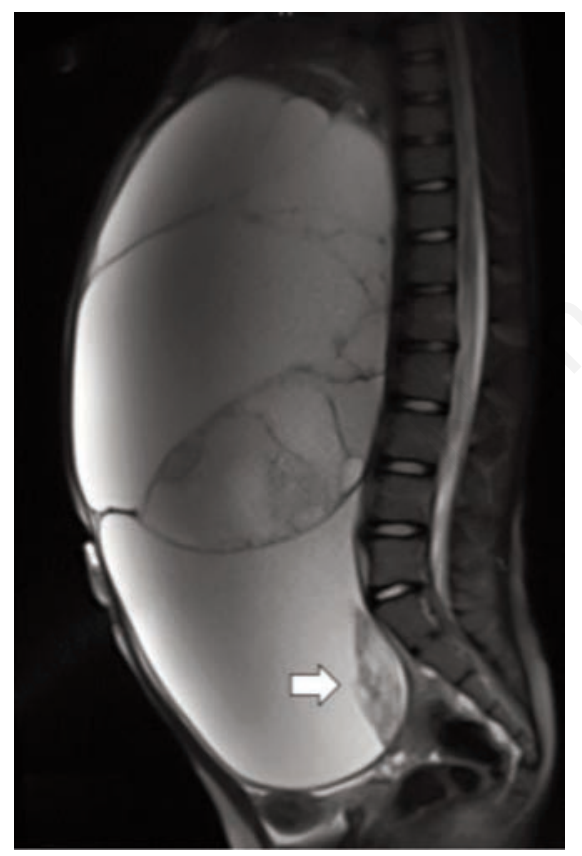

Figure 2. Preoperative MRI. There was a multilocular, high-intensity cyst with varying intensity on the T2-weighted images. Solid lesions had a lower intensity than did the internal solution on T2-weighted images. A structure that seems to be an oviduct was apparent on the bottom of the dorsal cyst (arrow). (which is defined as tumor limited to 1 ovary, capsule intact, no tumor on surface, and negative washings ${ }^{6}$ borderline MCA. Her postoperative course had no complications. Each tumor marker fell to within the normal range. The patient is doing well without recurrence or metastasis 5 years after the surgery.

\section{Discussion}

There is large amount of differential diagnosis in cystic abdominal masses in children. The possibility of diagnosis depends on which organ has cysts. Liver (mesenchymal hamartoma, biloma or parasitic cyst), biliary system (choledochal cyst or hydrops of gallbladder), spleen (congenital cyst), pancreas (congenital cyst, pseudocyst or cystadenoma), kidney (hydronephrosis, multicystic dysplastic kidney or multilocular cystic nephroma), adrenal grand (adrenal hemorrhage), gastrointestinal tract (mesenteric cyst, lymphangioma, duplication cyst, omental cyst or meconium pseudocyst), genitourinary (urachal cyst) and ovary (functional cyst, teratoma, dermoid cyst, cystadenoma, hematocolpos) may have cysts. In addition, there is the possibility of abscess, teratoma, necrotic or cystic changes in tumors, cere- brospinal fluid pseudocyst or sacrococcygeal teratoma. ${ }^{7}$

Ovarian tumors occur in 2.6 of 100,000 girls under 15 years of age, which represents $2 \%$ of all tumors in girls under 16 years of age. 1 Of those tumors, $67 \%$ are germ cell tumors, and $10-17 \%$ are epithelial tumors such as MCA or SCA.2,3 Epithelial tumors are mainly diseases of women in their $30 \mathrm{~s}$ and $50 \mathrm{~s} .{ }^{8}$

Most ovarian epithelial tumors are cystadenomas, $75 \%$ of which are serous and $25 \%$ of which are mucinous. 4,5 Among the epithelial tumors, mucous tumors have sticky gelatinous fluid and sometimes exhibit a multilocular large mass. Microscopically, cysts are formed by the epithelium with tall columnar mucinous differentiation. 9 Ovarian epithelial tumors in children are usually unilateral, and $12 \%$ are malignant. 10

There is a concept of borderline tumors in ovarian epithelial tumors, including MCA and SCA. An ovarian mucinous borderline tumor was first described in a study from the Cleveland Clinic in 1955.11 Thereafter, in the classification of the Cancer Committee of FIGO in 1971 and in addition to benign cystadenoma and cystadenocarcinoma, cystadenoma with proliferating activity of the epithelial cells and nuclear abnormalities but without infiltrative destructive growth (low-potential

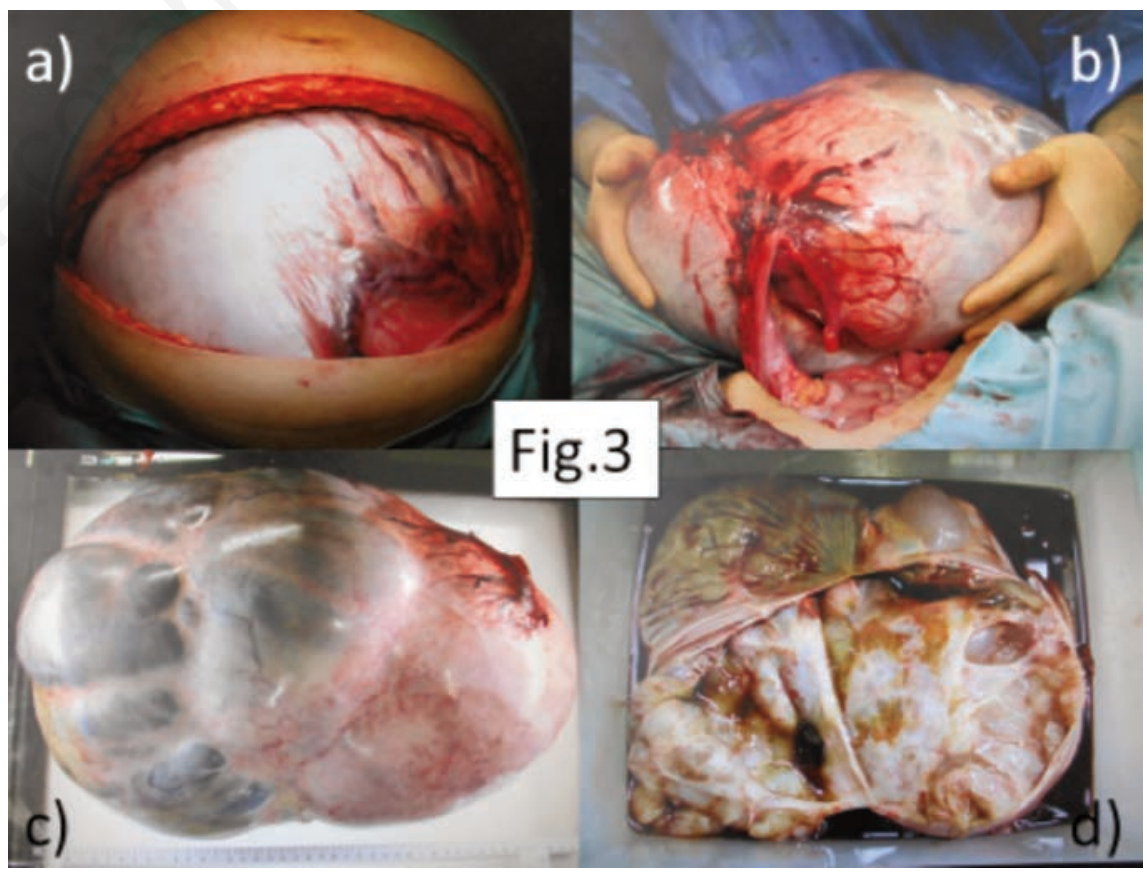

Figure 3. Intraoperative findings. a) Giant cysts with dilated vessels presented at laparotomy. b) The left oviduct was stretched and connected to the cyst. The ovarian parenchyma was ambiguous. c,d) The multilocular cystic tumor was $420 \times 220 \times 200 \mathrm{~mm}$ in size and $11.8 \mathrm{~kg}$ in weight. 
malignancy) was classified.12 Moreover, WHO (The World Health Organization) also used the concept of borderline malignant tumor and carcinoma of low malignancy in classifying ovarian tumors in 1973.13 Because borderline malignancy has a clearly better prognosis than that of cystadenocarcinoma, this appropriate classification is now, generally, pervasive.

Pathological diagnostic criteria for each group of mucinous tumors are as follows: 1) Benign MCA displays no nuclear layer stratification or stromal invasion. 2) The borderline malignant type of MCA displays nuclear stratification into 2 or 3 layers with no stromal invasion. 3) MCA displays stromal invasion or nuclear stratification of more than three layers. ${ }^{14,15}$

At pre- or intraoperation, the presence of a 'mural nodule' is helpful for classifying the tumor in some cases. A mural nodule is a several-centimeter protuberance of yellow, red or brown colored features, 16 and it consists of three types: (I) reactive sarcomatoid morphology, (II) undifferentiated cancer bud, and (III) a sarcoma nodule. Although type III follow the malignant course, type I has no effect on prognosis and type II also does not affect prognosis unless membrane damage occurs. ${ }^{17}$ Because ovarian borderline MCA can have multilocular cysts containing any type of nodule, we have to distinguish them. ${ }^{18,19}$ The features of type III are irregular shape, rough surface, wide basement, nonuniform internal echo and no blood flow. ${ }^{20}$ However, based on the study of 123 mucinous ovarian tumors, Moro et al. ${ }^{21}$ argued mural nodule is typical in endocervical-type borderline MCA. In this case, the nodules having wide basement and nonuniform internal echo was pathologically type I and the tumor was borderline MCA.

CA-125 is usually elevated during the growth of epithelial tumors. In ovarian tumor, Serous tumors tend to show elevated serum CA125.22 CA-19-9 levels rise when rupture, inflammation or adhesion occurs due to the large tumor. Several papers report that there is a significant correlation between tumor size and serum CA 19-9 values. ${ }^{23}$ Although Cho et al. ${ }^{23}$ reported that there was a significant correlation between serum CA 19-9 levels and the pathological types (borderline or malignancy), Kelly et al. ${ }^{24}$ denied the correlation. Therefore, the correlation between them is controversial.

Although the final diagnosis is guided by the pathological findings, intraoperative specimen diagnosis is often misleading. In this case, the intraoperative diagnosis was an adenoma, but the pathological diagnosis was changed to a borderline tumor.

Highly malignant lesions are newly dis- covered by adding cutouts after surgery. It seems to be more difficult to determine an appropriate intraoperative pathological diagnosis for MCA than it is for SCA because mucinous tumors tend to be larger. ${ }^{25}$ In the literature to date, the sensitivity of intraoperative diagnosis in the borderline group is as low as $44.4 \%$ to $50.0 \%$, indicating that accurate intraoperative specimen diagnosis is difficult. $25-29$

In adult stage I borderline MCA, the recurrence rate is $6 \% .30$ The 5-year survival rate is $98 \%$, and the 10 -year survival rate is $96 \%$ when appropriate surgery is performed. ${ }^{14}$ However, $92.8 \%$ of these cases are stage I, and there have been no reports of borderline MCA with metastasis. ${ }^{30}$ Even if borderline MCA is missed, the prognosis is almost always good. There is no problem if you can appropriately diagnose only MCA. 29

On the other hand, it was reported that death due to adjuvant therapy has more risk than natural cancer death in patients with stage I borderline MCA. Therefore, oophorectomy or salpingo-oophorectomy is necessary and sufficient. 18 With regard to the biopsy of the contralateral ovary, it is not preferable because of the potential for abdominal adhesion or damage to the normal ovary. ${ }^{31}$ Hence, our treatment was appropriate for this case. However, there are diverse opinions about the exploration of the contralateral healthy ovary.

Ottesen et al. 32 reported that the percentage of borderline malignancy and carcinoma adult giant ovarian mucinous tumors was $24 \%$, and there were no giant cases with metastasis. Mucinous tumors more rarely invade the ovary than do serous tumors. Therefore, symptoms other than abdominal distension are less likely to present in mucinous tumors.

In giant tumor cases, resection of the tumor may lead to some changes in respiration and circulation due to an increase in venous return, migration of thrombus or reexpansion of the lung. ${ }^{32}$ Therefore, pre- or intraoperative drainage is often considered. However, if the tumor has malignant potential, drainage of the cyst may lead to abdominal dissemination, and there is also the possibility of causing adhesion in the peritoneal cavity. Although recurrence of ovarian mucinous tumors is extremely rare, it has been reported that intraoperative rupture clearly affects recurrence.33,34 Therefore, achieving en bloc resection with no membrane damage is important. There was no significant difference in the incidence of complications when comparing cases of primary resection and resection with pre- and intraoperative drainage. We consider that if a tumor has malignant potential, primary resection by laparotomy is recommended. In our case, we performed a primary resection without drainage based on the possibility of malignancy because there were lesions suspected of mural nodules in the preoperative US and MRI findings. 32

In previous reports, a possibility of recurrence until 20 years later has been reported; therefore, long-term follow-up is required. ${ }^{35}$ We also plan to continue longterm follow-up in this case.

In adults, over $10 \mathrm{~kg}$ is defined as a giant ovarian tumor. ${ }^{36}$ The maximum ovarian MCA that was previously reported was $164 \mathrm{~kg}$ in adults, ${ }^{37}$ and $7.86 \mathrm{~kg}$ in patients aged 15 years or less. ${ }^{19}$ Therefore, this case is the maximum-sized tumor in children.

\section{Conclusions}

In ovarian mucinous tumors, prognosis is favored even in a giant case, except for MCA.

There may be options for drainage before or during surgery to avoid unfavorable perioperative events, such as fluctuations in respiration and circulation. However, if the tumor is possibly malignant, primary oophorectomy or salpingooophorectomy is recommended. It is extremely important to not miss the findings that are suggestive of malignancy, such as multilocular cysts with mural nodules or high values of tumor markers.

\section{References}

1. Lindfors O. Primary ovarian neoplasms in infants and children: a study of 81 cases diagnosed in Finland and Sweden. Ann Chir Gynaecol Fenn Suppl 1971; 177:1-66.

2. Freud E, Golinsky D, Steinberg RM, et al. Ovarian masses in children. Clin Pediatr (Phila) 1999;38:573-7.

3. Helmrath MA, Shin CE, Warner BW. Ovarian cysts in the pediatric population. Semin Pediatr Surg 1998;7:19-28.

4. Parmentier B, Vaz E, ChabaudWilliamson M, et al. Mucinous cystadenoma arising 3 years after ovarian-sparing surgery for mature teratoma in a child. J Pediatr Surg 2010;45:9-12.

5. Karaman A, Azil MN, Boduroglu EC, et al. A huge ovarian mucinous cystadenoma in a 14-year-old premenarchal girl: review on ovarian mucinous tumor in premenarchal girls. J Pediatr Adolesc Gynecol 2008;21:41-4.

6. Zeppernick F, Meinhold-Heerlein I. The 
new FIGO staging system for ovarian, fallopian tube, and primary peritoneal cancer. Arch Gynecol Obstet 2014;290:839-42.

7. Sandra L, Kristen B, Roger K, et al. Giant cystic abdominal masses in children. Pediatr Radiol 2005;35:1277-88.

8. Brown MF, Hebra A, McGeehin K, et al. Ovarian masses in children: a review of 91 cases of malignant and benign masses. J Pediatr Surg 1993;28:930-3.

9. Sri Paran T, Mortell A, Devaney D, et al. Mucinous cystadenoma of the ovary in perimenarchal girls. Pediatr Surg Int 2006;22:224-7.

10. Abell MR, Holtz F. Ovarian neoplasms in childhood and adolescence. II. Tumors of non-germ cell origin. Am J Obstet Gynecol 1965;93:850-66.

11. Fisher ER, Kreiger JS, Skirpan PJ. Ovarian cystoma: Clinicopathological observations. Cancer 1955;8:437-45.

12. FIGO. International Federation of gynecology and Obstetrics. Classification and staging of malignant tumours in the female pelvis. Acta Obstet Gynecol Scand 1971;50:1-7.

13. Serov SF, Scully RE, et al. International histologic Classification of tumours. No. 9. Histological Typing of Ovarian Tumours. World Health Organization, Geneva 1973.

14. Hart WR, Norris HJ. Borderline and malignant mucinous tumours of the ovary: histologic criteria and clinical behavior. Cancer 1973;31:1031-45.

15. Morris HB, La Vecchia C, Draper GJ. Malignant epithelial tumors of the ovary in childhood: a clinicopathological study of 13 cases in Great Britain 1962-1978. Gynecol Oncol 1984;19:290-7.

16. Lee, KR, Tavassoli FA, Prat J, et al. Surface epithelial-stromal tumors. WHO classification of tumours. In: Tavasolli FA, Stratton MR, eds. Pathology and Genetics. Tumours of the breast and female genital organs. Lyon: IARC Press; 2003. pp 117-45.
17. Provenza C, Young RH, Prat J. Anaplastic carcinoma in mucinous ovarian tumors: a clinicopathologic study of 34 cases emphasizing the crucial impact of stage on prognosis, their histological spectrum, and overlap with sarcomatous mural nodules. Am J Surg Pathol 2008;32:383-9.

18. Hart WR. Borderline epithelial tumors of the ovary. Modern pathology 2005; 18:33-50.

19. Cevik M, Guldur ME. An extra-large ovarian mucinous cystadenoma in a premenarchal girl and a review of the literature. J Pediatr Adolesc Gynecol 2013;26:22-6.

20. Zhang Y, Yuan Z, Sun K, Li P. Ultrasonic and pathological characteristics of ovarian mucinous cystic tumors with malignant mural nodules: Two cases report. Medicine 2017;96:45.

21. Moro F, Zannoni GF, Arciuolo D, et al. Imaging in gynecological disease (11): clinical and ultrasound features of mucinous ovarian tumors. Ultrasound Obstet Gynecol 2017;50:261-70.

22. Nakagawa N, Koda H, Nitta N, et al. Reactivity of CA19-9 and CA125 in histological subtypes of epithelial ovarian tumors and ovarian endometriosis. Acta Med Okayama 2015;69:227-35.

23. Cho HY, Kyung MS. Serum CA19-9 as a predictor of malignancy in primary ovarian mucinous tumors: a matched case-control study. Med Sci Monit 2014;20:1334-9.

24. Kelly PJ, Archbold P, Price JH, et al. Serum CA19.9 levels are commonly elevated in primary ovarian mucinous tumours but cannot be used to predict the histological subtype. J Clin Pathol 2010;63:169-73.

25. Rose PG, Rubin RB, Nelson BE, et al. Accuracy of frozen-section (intraoperative consultation) diagnosis of ovarian tumors. Am J Obstet Gynecol 1994;171:823-6.

26. Rotterdam H, Slavutin L. Frozen section diagnosis of serous epithelial tumors of the ovary. Am J Diagn Gynecol Obset 1979;1:89-94.

27. Bastos AC, Salvatore CA, Faria RM. Frozen section biopsy of ovarian neoplasms. Int $\mathrm{J}$ Gynaecol Obset 1983;21:103-10.

28. Twaalfhoven FC, Peters AA, Trimbos JB, et al. The accuracy of frozen section diagnosis of ovarian tumors. Gynecol Oncol 1991;41:189-92.

29. Obiakor I, Maiman M, Mittal K, et al. The accuracy of frozen section in the diagnosis of ovarian neoplasms. Gynecol Oncol 1991;43:61-3.

30. Lee KR, Scully RE. Mucinoustumors of the ovary: a clinicopathologic study of 196 borderline tumors (of intestinal type) and carcinomas, including an evaluation of 11 cases with 'pseudomyxoma peritonei'. Am J Surg Pathol 2000;24:1447-64.

31. Iwasaki M, Taira K, Kobayashi $H$, Saiga T. Ovarian mucinous cystadenoma of borderline malignancy in a premenarchal girl. J Pediatr Adlesc Gynecol 2010;23:119-23.

32. Ottesen M, Rose M. Giant ovarian tumor masked by obesity. Acta Obstet Gynecol Scand 1994;73:349-51.

33. Hernandez E, Rosenshein NB, Parmley TH. Mucinous cystadenoma in a premenarchal girl. South Med J 1982;75:1265-7.

34. Vizza E, Galati GM, Corrado G, et al. Voluminous mucinous cystadenoma of the ovary in a 13-year-old girl. J Pediatr Adolesc Gynecol 2005;18:419-22.

35. Link CJ Jr., Reed E, Sarosy G, Kohn EC. Borderline ovarian tumors. Am J Med 1996;101:217-25.

36. Hori M, Imai A, Sugiyama $T$, et al. Massive ovarian serous cystadenoma with uneventful postoperative recovery. Gynecol Obstet Invest 1991;32:245-6.

37. Spohn AE. Multicystic ovarian tumor weighing 328 lb. Tex Med J 1905;1:273-4. 